\title{
TU/e EmonOWEN

\section{Surface plasmon at a metal-dielectric interface with an epsilon-near-zero transition layer}

\section{Citation for published version (APA):}

Roccapriore, K., Bozhko, A., Nazarikov, G., Drachev, V., \& Krokhin, A. (2021). Surface plasmon at a metaldielectric interface with an epsilon-near-zero transition layer. Physical Review B, 103(16), [L161404]. https://doi.org/10.1103/PhysRevB.103.L161404

DOI:

10.1103/PhysRevB.103.L161404

Document status and date:

Published: $13 / 04 / 2021$

\section{Document Version:}

Publisher's PDF, also known as Version of Record (includes final page, issue and volume numbers)

\section{Please check the document version of this publication:}

- A submitted manuscript is the version of the article upon submission and before peer-review. There can be important differences between the submitted version and the official published version of record. People interested in the research are advised to contact the author for the final version of the publication, or visit the $\mathrm{DOI}$ to the publisher's website.

- The final author version and the galley proof are versions of the publication after peer review.

- The final published version features the final layout of the paper including the volume, issue and page numbers.

Link to publication

\section{General rights}

Copyright and moral rights for the publications made accessible in the public portal are retained by the authors and/or other copyright owners and it is a condition of accessing publications that users recognise and abide by the legal requirements associated with these rights.

- Users may download and print one copy of any publication from the public portal for the purpose of private study or research.

- You may not further distribute the material or use it for any profit-making activity or commercial gain

- You may freely distribute the URL identifying the publication in the public portal.

If the publication is distributed under the terms of Article 25fa of the Dutch Copyright Act, indicated by the "Taverne" license above, please follow below link for the End User Agreement:

www.tue.nl/taverne

Take down policy

If you believe that this document breaches copyright please contact us at:

openaccess@tue.nl

providing details and we will investigate your claim. 


\title{
Surface plasmon at a metal-dielectric interface with an epsilon-near-zero transition layer
}

\author{
Kevin Roccapriore $\odot,{ }^{1,2}$ Andrey Bozhko, ${ }^{1}$ Gleb Nazarikov $\odot,{ }^{3,4}$ Vladimir Drachev $\odot,{ }^{1,3,{ }^{*}}$ and Arkadii Krokhin $\oplus^{1, \dagger}$ \\ ${ }^{1}$ Department of Physics, University of North Texas, P.O. Box 311427, Denton, Texas 76203, USA \\ ${ }^{2}$ Center for Nanophase Materials Sciences, Oak Ridge National Laboratory, Oak Ridge, Tennessee 37831, USA \\ ${ }^{3}$ Skolkovo Institute of Science and Technology, 3 Nobel Street, Moscow 143026 Russia \\ ${ }^{4}$ Department of Electrical Engineering, Eindhoven University of Technology, $5600 \mathrm{MB}$ Eindhoven, The Netherlands
}

(Received 3 December 2020; accepted 30 March 2021; published 13 April 2021)

\begin{abstract}
A metal-dielectric interface is usually modeled by a zero-width layer, where dielectric permittivity suffers a discontinuity jump. In reality, there is a narrow transition region, which includes an epsilon-near-zero (ENZ) layer. We show that due to a continuous dielectric function the dispersion of the surface plasmon is modified. The energy of the surface plasmon radiates through the ENZ layer, giving rise to additional radiative losses. Direct evidence of the effects related to the transition layer is given by the observation of plasmonic resonance in the presence of a strong electric field normal to the metal film. The electric field affects the electron density within the transition layer that leads to a measurable shift of the plasmonic resonance.
\end{abstract}

DOI: 10.1103/PhysRevB.103.L161404

Nanophotonics research explores ways to concentrate electromagnetic fields within the subwavelength regions, and the metamaterials provide much needed unconventional material properties to assist with that [1]. The prospect of accumulating energy and thus achieving an unprecedented field enhancement at the nanoscale drives the development of optoelectronic devices and near-field imaging and conceives new applications for tumor treatment and solar cell design [2-4]. The interaction of light with surface plasmon polaritons (SPPs) strongly enhances linear and nonlinear effects $[5,6]$, however, there is a certain downside to plasmon-enabled processes [7]. It is known that scaling down the physical system raises questions regarding SPP radiation [8,9], stability $[10,11]$, nonlocal response [11-14], and quantum tunneling effects $[15,16]$.

Due to the high concentration of electromagnetic energy, the SPP may effectively probe the surface itself [17] and also enhance the wave-matter interaction. However, because of the Joule and radiative losses and Landau damping, the observed level of enhancement turns out to be much weaker than the theoretical expectations $[8,9,18]$. At the same time, highly localized thermal losses find many useful applications [19].

Here, we present one more fundamental reason for low efficiency, related to the inevitable radiative losses of the SPP. A propagating SPP radiates energy through the epsilon-nearzero (ENZ) region formed by spill-out electrons and surface roughness $[16,20]$. While the thickness of this layer is only a few $\mathrm{nm}$, it affects the spatial distribution of energy, dispersion, and attenuation of the SPP. Thus, it turns out that the SPP is naturally a leaky mode even when it propagates along a plane boundary between a metal and dielectric.

\footnotetext{
*vladimir.drachev@unt.edu

${ }^{\dagger}$ Arkadii.Krokhin@unt.edu
}

The existence of a SPP as an eigenmode with eigenfrequencies lying below the plasma frequency, $\omega(k)<\omega_{p}$, is due to the possibility of matching the tangential components of the electric and magnetic fields at a metal-dielectric interface where the dielectric function suffers a discontinuity from $\epsilon(\omega)<0$ in the metal to $\epsilon_{d}>0$ in the dielectric. However, a discontinuous transition from a metal to a dielectric is a mathematical idealization of a rather sharp but continuous change of the dielectric function. For some electromagnetic phenomena an ideally sharp boundary turns out to be a valid approximation and for this reason the Fresnel formulas usually give adequate results. Landau and Lifshitz [21] refer to the validity of Fresnel formulas by mentioning that "the derivation of Fresnel formulae, on the other hand, is based on the use of the boundary conditions, and assumes that the thickness $\delta$ of the transition layer is small compared with wavelength $\lambda . "$

While the condition $\delta \ll \lambda$ is usually satisfied, it is of principal importance to account for the finite-width transition layer between the metal and dielectric because in this case the transition layer contains a region with ENZ. At atomically flat surfaces the transition layer of thickness $\delta \sim 1-2 \mathrm{~nm}$ is formed by spill-out electrons that form a cloud due to quantum fluctuations in the Fermi sea of bulk conduction electrons [22]. At a rough surface, a much thicker transition layer exists as a result of averaging of the electron density over the fluctuating profile of metal peaks and dips.

While the thickness of the transition layer may be less than $1 \mathrm{~nm}$, a macroscopic description with an effective dielectric function remains valid. The calculation of this effective dielectric function requires a microscopic approach, which accounts for the effects of spatial dispersion [13,23].

Because of the presence of the transition layer the properties of the metal-dielectric structure are described by a continuous function $\epsilon(z)$ of the normal to the interface coordinate z. As shown in the Supplemental Material [24], the 
main results for the spectrum of SPP are not sensitive to the specific form of $\epsilon(z)$ (see also the discussion in Ref. [25]), which should be topologically equivalent to a kink. A simple choice is

$$
\epsilon(z)=\epsilon_{d}+\frac{\epsilon(\omega)-\epsilon_{d}}{1+\exp (z / \delta)} \rightarrow \begin{cases}\epsilon_{d}, & z \rightarrow \infty \\ \epsilon(\omega), & z \rightarrow-\infty\end{cases}
$$

At the critical point $z_{0}=\delta \ln \left[-\epsilon(\omega) / \epsilon_{d}\right]$ the dielectric function vanishes, if dissipation in the metal is neglected. This point is a point of singularity in the wave equation for the $H$ component of the $p$-polarized wave with components $E_{x}, E_{z}, H_{y}=H(z) \exp (i k x-i \omega t)$,

$$
\frac{d}{d z}\left(\frac{1}{\epsilon(z)} \frac{d H}{d z}\right)+\left(\frac{\omega^{2}}{c^{2}}-\frac{k^{2}}{\epsilon(z)}\right) H(z)=0 .
$$

The logarithmic singularity of the solution to this equation near $z=z_{0}$ is discussed in Ref. [21]. The excitation of bulk transverse magnetic (TM) waves in a transversely inhomogeneous plasma with a $z$-dependent plasma frequency was studied in detail in Refs. [26,27].

The wave equation (2) contains an obvious small parameter $k \delta \sim \delta / \lambda \ll 1$. However, a standard perturbation theory over $k \delta$ is not valid because of the divergence at $z=z_{0}$. To overcome this difficulty, we apply a Fourier transformation over coordinate $z$ and obtain the following integral equation for the Fourier component $h(p)$ :

$$
\frac{\omega^{2}}{c^{2}} h(p)=\int_{-\infty}^{+\infty}\left(k^{2}+p p^{\prime}\right) \eta\left(p-p^{\prime}\right) h\left(p^{\prime}\right) d p^{\prime} .
$$

Here, the kernel $\eta(p)=(2 \pi)^{-1} \int_{-\infty}^{\infty} e^{-i p z} / \epsilon(z) d z$ is Hermitian in the dissipationless medium $[\operatorname{Im} \epsilon(z)=0]$ if the dielectric function does not vanish along the path of integration. Then, the eigenfrequency $\omega(k)$ is real. For the model of a discontinuous medium $(\delta=0)$, Eq. (3) has an obvious solution giving the dispersion for the SPP,

$$
\omega_{0}^{2}(k)=k^{2} c^{2}\left(\frac{1}{\epsilon_{d}}-\frac{1}{|\epsilon(\omega)|}\right) .
$$

The symmetry of Eq. (3) changes drastically if integration within the transition layer goes through the point $z_{0}$ where the dielectric function vanishes. Bypassing the singularity at $z_{0}$ gives an imaginary contribution to $\eta(p)$ even if $\epsilon(z)$ is real,

$$
\eta(p)=\frac{1}{2 \pi} \int_{-\infty}^{+\infty} \frac{e^{-i p z}}{\epsilon(z)} d z-\frac{i}{2} \operatorname{Res}_{z=z_{0}} \frac{e^{-i p z}}{\epsilon(z)} .
$$

The second term here clearly breaks the hermiticity of the kernel in Eq. (3), resulting in the complex eigenvalues $\omega(k)=$ $\omega_{0}(k)+\omega^{\prime}(k)-i \omega^{\prime \prime}(k)$. Once the singularity has been integrated out, the integral equation (3) with the kernel (5) can be solved by perturbation theory (see the Supplemental Material [24]). Omitting details, we give here the final result for the linear over $\delta$ corrections to the unperturbed plasmonic spectrum $\omega_{0}(k)$, which defines the shift of the resonant frequency $\omega^{\prime}$ and radiative decay $\omega^{\prime \prime}$,

$$
\begin{gathered}
\frac{\omega^{\prime}(k)}{\omega_{0}(k)}=-\delta \frac{k^{2}+\kappa_{d}^{2}}{\kappa_{m}-\kappa_{d}} \ln \left|\frac{\epsilon(\omega)}{\epsilon_{d}}+1\right|, \\
\frac{\omega^{\prime \prime}(k)}{\omega_{0}(k)}=\pi \delta \frac{k^{2}+\kappa_{d}^{2}}{\kappa_{m}-\kappa_{d}} .
\end{gathered}
$$

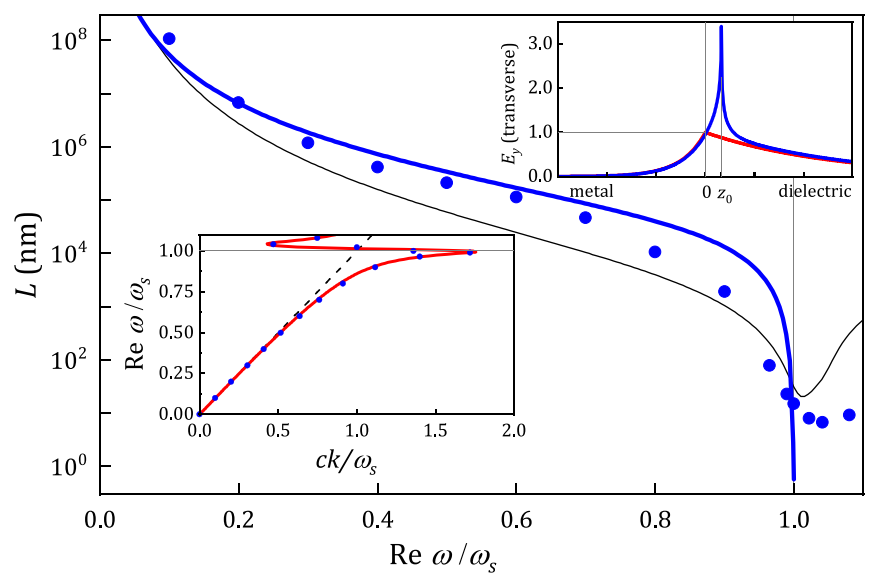

FIG. 1. Decay of SPP propagating along the silver-vacuum interface. The decay is given in terms of propagation length $L=1 / 2 \operatorname{Im} k$ as a function of normalized frequency $\omega / \omega_{s}$. The thickness of the transition layer $\delta=0.5 \AA$. The blue dots are the radiative decay $L_{\text {rad }}$ calculated numerically from Eq. (2) and the blue line is the radiative decay calculated in the linear approximation $L_{\text {rad }} \approx v_{g} / 2 \operatorname{Im} \omega$ with $\operatorname{Im} \omega=-\omega^{\prime \prime}$ given by Eq. (7). Decay due to Joule losses in silver with $\omega_{p}=9.1 \mathrm{eV}$ and electron relaxation frequency $v=0.048 \mathrm{eV}$ (room temperature) is shown by the black line. Left inset: Dispersion relation of SPP calculated for the model of a discontinuous interface ( $\delta=0$, red line) and for the transition layer $(\delta=0.5 \AA$, blue dots). Right inset: Enhancement of the transverse, $E_{y}$, component of the electric field of SPP near the ENZ point (blue) as compared to the structure without the transition layer (red).

Here, $\kappa_{d}=\sqrt{k^{2}-\epsilon_{d} \omega^{2} / c^{2}}\left[\kappa_{m}=\sqrt{k^{2}+|\epsilon(\omega)| \omega^{2} / c^{2}}\right]$ is the inverse decay length of the fields of an unperturbed plasmon in the dielectric (metal).

The imaginary correction to the eigenfrequency value means that the SPP loses energy even if the metal is lossless, i.e., $\operatorname{Im} \epsilon(\omega)=0$. This mechanism of plasmonic decay is in some sense similar to Landau damping [7,11], since neither is due to the electron collisions in the metal. However, the decaying SPP radiates energy to the bulk plasmon through the ENZ region, unlike the energy transfer from the bulk plasmon to resonant electrons that occurs in the mechanism of Landau damping $[20,25]$.

To compare the contribution of radiation and dissipative losses for SPP propagating along a silver-vacuum interface, the propagation lengths $L=1 / 2 \operatorname{Im} k$ due to different mechanisms are plotted in Fig. 1. The radiative losses (7) calculated in the linear approximation over $k \delta$ diverge near the frequency of plasmonic resonance $\omega_{s}=\omega_{p} / \sqrt{1+\epsilon_{d}}$, where the group velocity $v_{g}=d \omega_{0} / d k \rightarrow 0$. Therefore, the propagation length $L_{\mathrm{rad}}=1 / 2 \operatorname{Im} k \approx\left|v_{g}\right| / 2 \operatorname{Im} \omega$ (blue line) vanishes as $\left(\omega_{s}-\omega\right)^{3}$ near the resonance. However, the propagation length calculated numerically from Eq. (2) remains finite at $\omega_{s}$ (blue dots). Apart from the narrow region near the plasmonic resonance the linear approximation based on Eq. (7) is quite close to the exact numerical result. The radiative losses are calculated for a very thin transition layer, $\delta=0.5 \AA$, which corresponds to an atomically flat silver surface. In a typical thin film used in optical experiments the surface roughnesses form a much thicker transition layer with $\delta$ of the order of 
a few nanometers. According to Eq. (7), the radiative losses in such samples are an order of magnitude higher. While the thickness of the transition layer is very narrow, the radiative losses are comparable to the dissipative losses shown by the black line in Fig. 1. Radiative and dissipative losses give additive contributions to the spatial attenuation coefficient $1 / L$ of SPP, $1 / L=1 / L_{\text {diss }}+1 / L_{\text {rad }}$. The low efficiency of plasmonic devices is usually associated with dissipative losses $[8,9,19]$. It, however, may be due to the essential contribution of radiative losses through the transition layer.

The thickness $\delta$ and spatial distribution of the electron charge near the metal surface can be controlled by an external dc field $\mathbf{E}$ applied perpendicular to the metal-dielectric interface. In particular, the position of the singular point $z_{0}$ relative to the interface defines not only the radiative losses but also the enhanced amplitude of the electromagnetic field of SPP with components $\left(E_{x}, E_{y}, 0\right),(0,0, H)$ near the surface as demonstrated in the right inset to Fig. 1 for the component $E_{y}$. This additional enhancement of the SPP field contributes to the sensitivity of surface-enhanced Raman spectroscopy (SERS), which is proportional to $E^{4}$. The advantages of field enhancement important for different applications were recently considered in a review [28]. It is important to note that the Joule losses in a metal always reduce the strength of the fields of the SPP [19]. Unlike this, the radiative losses are accompanied by field enhancement in the ENZ layer. However, the amplitude of field enhancement at $z=z_{0}$ can be reduced by the effects related to spatial dispersion in metal conductivity—Landau damping [7,29,30]. The most important property which is affected by the external electric field is the angular distribution of intensity in the reflected beam. The latter property will be used for an experimental demonstration of the existence of the transition layer and its influence on the spectrum of SPP.

In the lowest approximation the width of the transition layer changes linearly with $\mathbf{E}$,

$$
\delta(\mathbf{E})=\delta_{0} \pm \alpha_{ \pm} E,
$$

where $\delta_{0}$ is the bare width of the transition layer without an applied electric field. The coefficients $\alpha_{ \pm}$characterize the rate of increase or decrease of $\delta$ with the strength of the applied field. These rates are different for the field pulling electrons out of the metal $\left(\alpha_{+}\right.$, positive bias) and in the metal $\left(\alpha_{-}\right.$, negative bias). The width $\delta(\mathbf{E})$ remains positive for a metal-dielectric interface, for any direction of $\mathbf{E}$.

It follows from Eq. (6) that the shift $\omega^{\prime}$ is negative. While for the dissipationless metal $[\operatorname{Im} \epsilon(\omega)=0]$ the logarithm formally changes its sign passing through the resonance, the corrections $\omega^{\prime}$ and $\omega^{\prime \prime}$ diverge at the resonance, i.e., the perturbation theory fails. For a real metal with $\operatorname{Im} \epsilon(\omega)>0$ the shift $\omega^{\prime}$ remains negative. A redshift in the SPP resonance in $\mathrm{Mg}$ and $\mathrm{Al}$ was observed a long time ago in experiments with electron energy losses [31]. Later, this redshift was theoretically explained by the inhomogeneous distribution of conduction electrons across a metal-vacuum interface within the jellium model [32]. A redshift in the localized surface plasmon resonance in metal nanoparticles caused by spill-out electrons was predicted in Refs. [16,33,34] and experimentally confirmed for optical frequencies in Ref. [35]. However, (a)

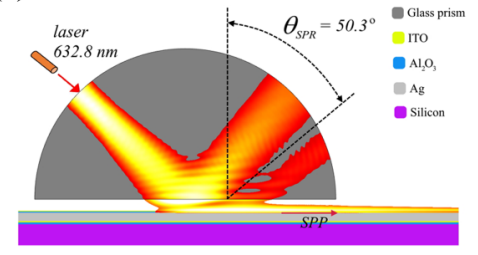

(b)

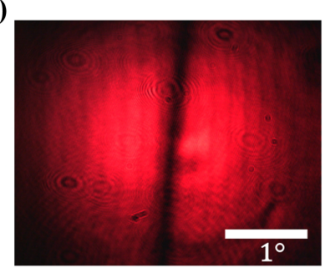

FIG. 2. A scheme of the experimental Otto configuration setup with the distribution of light intensity. (a) A 150-nm-thick silver sample is lying on a 50-nm ITO electrode. A second 15 -nm ITO electrode on the top is separated from the sample by a 20 -nm-thick aluminum oxide insulating layer. The device is fabricated on a silicon substrate covered by a 10 -nm-thick silicon-oxide layer. The width-controlled air gap is created by the spin-coating poly(methyl methacrylate) (PMMA). A hemispherical glass prism is placed on top. Distribution of light intensity obtained from COMSOL simulation. The silver-air interface is modeled by a transition layer Eq. (1) with $\delta=0.5 \AA$ A. (b) Experimentally observed map of reflected intensity. The black region between two red spots corresponds to the minimum in reflection caused by the SPP excitation.

for silver nanoparticles of size less than $10 \mathrm{~nm}$, a nonlocal contribution to electron conductivity near the metal surface, treated in a hydrodynamic approximation, leads to a strong blueshift, which overcomes the redshift [36].

Our experimental setup using the Otto configuration for the excitation of a SPP in a silver film is shown in Fig. 2. A SPP is excited by a $p$-polarized HeNe laser emitting at $\lambda=632.8 \mathrm{~nm}$ by attenuated total reflection. A silver film of thickness $150 \mathrm{~nm}$, that exceeds the skin depth of $25 \mathrm{~nm}$ in silver, can be considered as a semi-infinite sample. We used a custom-built setup which allows us to arbitrarily position and direct the laser beam onto the prism and simultaneously position a CCD to scan the reflectance of the laser beam as a function of angle $\theta_{\text {SPR }}$. A typical distribution of light intensity obtained by COMSOL simulation is illustrated by Fig. 2(a). The reflected intensity exhibits a profound minimum at the angle $\theta_{\mathrm{SPR}} \approx$ $50^{\circ}$ obtained from the matching of the wave vectors, $k(\omega)=$ $(2 \pi n / \lambda) \sin \theta_{\mathrm{SPR}}$, where $n=1.64$ is the index of refraction of the prism. The experimentally measured reflected intensity shown in Fig. 3 also has a deep minimum at $\theta_{\mathrm{SPR}}=50.3^{\circ}$ (see the leftmost curve at $\mathbf{E}=0$ ).

In the model of a sharp boundary the electric field $\mathbf{E}$ has no influence on the spectrum of SPP and the distribution of reflected light. Unlike this, the distribution of electron charge in the transition layer changes with $\mathbf{E}$ giving rise to the corrections (6) and (7). The field $\mathbf{E}$ was applied through the optically transparent and conductive indium tin oxide (ITO) electrodes (see Fig. 2) and the angular distribution of reflected light was measured for some discrete values of $\mathbf{E}$. The evolution of the angular distribution near the minimum of reflected intensity with increasing strength $E$ is shown in Fig. 3 for two opposite directions of $\mathbf{E}$. The shape of the minimum remains practically unchanged but the position of the minimum is shifted towards larger angles.

The angular shift $\Delta \theta$ is due to the redshift $\omega^{\prime}<0$. The expansion of the matching condition for the wave vectors $k\left(\omega_{0}+\omega^{\prime}\right)=\left(\omega_{0} / c\right) \sin \left(\theta_{\mathrm{SPR}}+\Delta \theta\right)$ is adjusted by a 
(a)

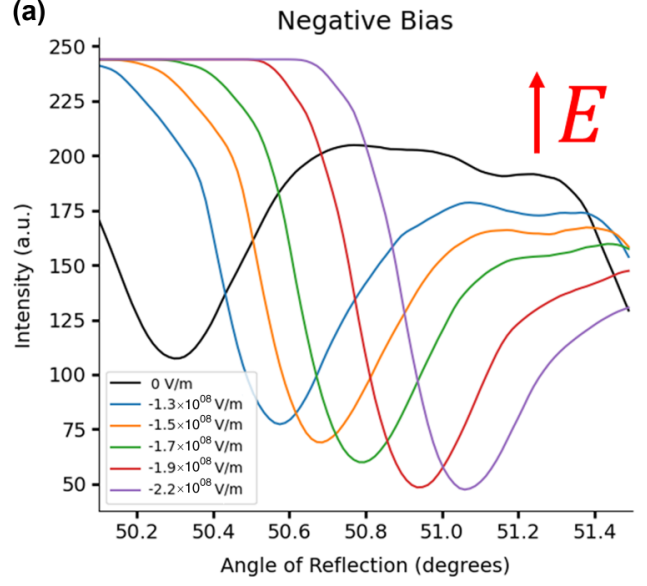

(b)

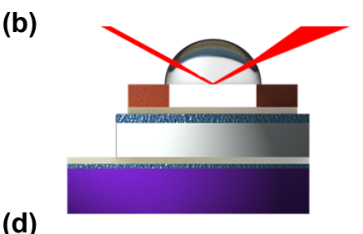

(d)

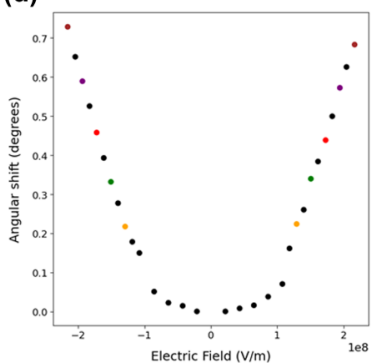

(c)

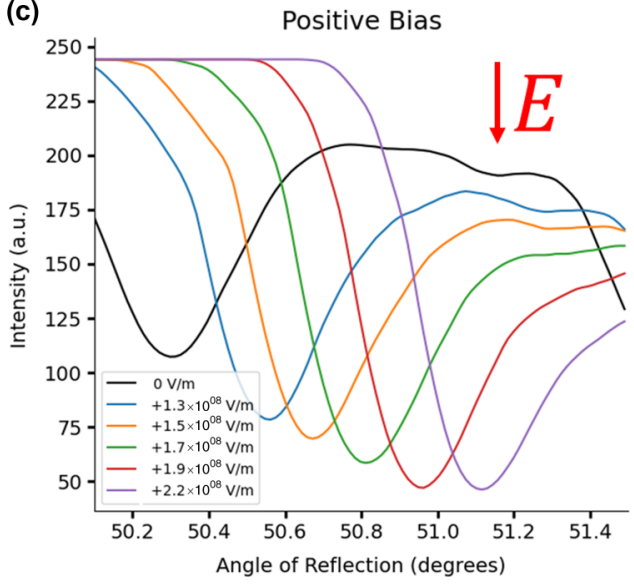

FIG. 3. Angular distribution of reflected light intensity measured at different strengths of the DC electric field. For the opposite directions of the electric field the position of the minimum is shifted towards larger angles when increasing the DC field strength. In (a), the field is directed up, while in (c) it is directed downward, with respect to the device geometry in (b). (d) illustrates angular shift $\Delta \theta$ vs electric field. This curve is plotted with smaller steps over the field strength $E$ than the curves showing an angular dependence of intensity.

positive shift,

$$
\Delta \theta=-\frac{c \omega^{\prime}}{\left|v_{g}\right| n \omega_{0} \cos \theta_{\mathrm{SPR}}} \sim \delta_{0} \pm \alpha_{ \pm} E>0 .
$$

This equation is valid near the resonance where the group velocity $v_{g}$ is negative, as shown in the inset to Fig. 1 . While the total shift is positive, the field-dependent part $\pm \alpha_{ \pm} E$ changes its sign when the field $\mathbf{E}$ is inverted. Since in the experiment only this part of $\Delta \theta$ is measured, one may expect a positive or negative shift depending on the direction of vector
E. However, only a positive angular shift for both directions of $\mathbf{E}$ was registered in our experiment.

The angular shifts are extracted from the sets of resonant curves in Fig. 3 obtained for negative and positive bias. In both cases the field-dependent shift turns out to be positive, exhibiting a practically symmetric dependence $\Delta \theta(-E) \approx \Delta \theta(E)$. Moreover, the shift $\Delta \theta$ exhibits a nonlinear dependence with strength $E$, as shown in the lower inset to Fig. 3 .

We suspect the reason for these disagreements is related to the screening effect produced by the upper ITO electrode. The conducting ITO plate, which is ignored in the derivation
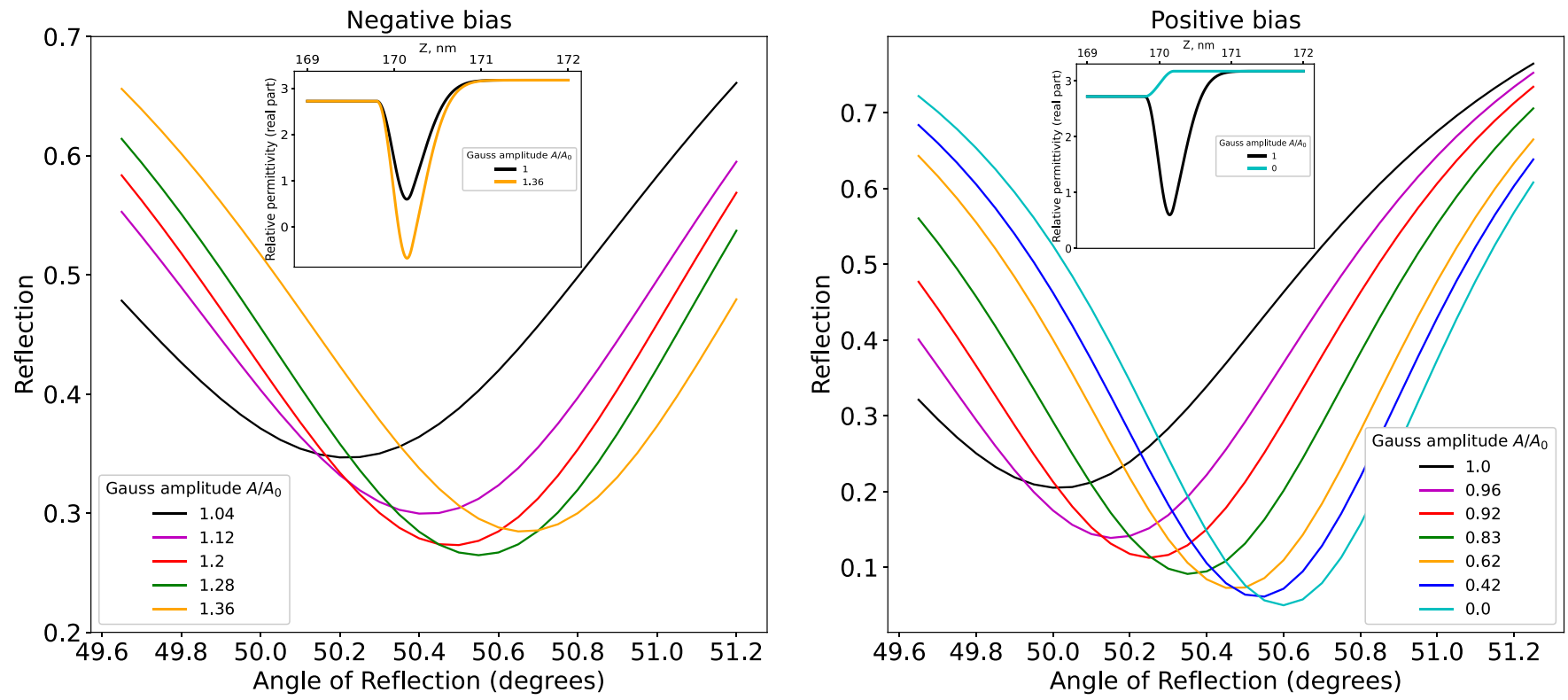

FIG. 4. Numerically simulated angular distribution of reflected light intensity at different strengths of the DC electric field. The always positive shift of the resonant minima with electric field strength obtained from the drift-diffusion model reproduces the experimental results of Fig. 3. The electric field changes from $0 \mathrm{~V}$ (black line) to its maximum value, corresponding to a bias of $\pm 5 \mathrm{~V}$. The profile of the relative dielectric permittivity across the ITO-alumina structure (shown in the insets) is modeled by a Gaussian function with a constant width of $0.5 \mathrm{~nm}$ and field-dependent amplitude $A=A(E)$ with $A_{0}=A(0)$. Insets: The profiles of the relative dielectric permittivity across the ITO-alumina structure for positive and negative bias. Coordinate $z$ is counted from the silver-alumina interface. 
of Eqs. (1)-(9), affects the dispersion and decay of SPP. The thickness of the $\mathrm{Al}_{2} \mathrm{O}_{3}$ layer insulating the silver film from the ITO plate is $20 \mathrm{~nm}$, which is comparable to the decay length of the SPP in the dielectric, $1 / \kappa_{d}$. Electromagnetic coupling between silver and ITO leads to the formation of a gap plasmon propagating inside a capacitor with plates of very different conductivities. It is known that very asymmetric plasmonic structures have specific peculiarities that do not exist in symmetric structures [37]. To the best of our knowledge, gap-plasmon propagation in an asymmetric system has yet to be considered. The clouds of spill-out electrons of the ITO electrode and silver film do not overlap, i.e., there is no electron tunneling through the insulating layer. Tunneling modifies gap-plasmon resonance between two metal nanospheres separated by a distance of less than $1 \mathrm{~nm}[38]$.

Because of the low electron concentration in ITO the electric field $\mathbf{E}$ renders a stronger effect on the distribution of conduction electrons in the upper plate than in the silver film. An analysis of gap-plasmonic resonance in the system shown in Fig. 2 including an ITO electrode was done numerically using COMSOL software. The results obtained for a simultaneous solution of the drift-diffusion model for charge carriers and Maxwell's equations are plotted in Fig. 4. More details of the model are given in Ref. [24].

These results reproduce the behavior of the experimental resonant curves, namely, a positive shift $\Delta \theta$ for both directions of the applied electric field and a nonlinear, slightly asymmetric dependence $\Delta \theta(\mathbf{E})$. While we do not know exactly the details of the transition layers in the silver film and ITO electrode, we may claim that the values of the parameters selected for the model are close to their real values. A positive shift is numerically reproduced only if the parameters of the model fit a narrow interval. If the parameters of the transition layers deviate by more than $\sim 10 \%$ from the selected ones, the shift $\Delta \theta$ changes its sign with the direction of the applied electric field $\mathbf{E}$. We hope that further development of the model and advancement in experimental techniques will lead to better accuracy in the determination of the parameters of real metal surfaces.

In summary, the radiative nature of a SPP propagating along a realistic metal-dielectric interface where a narrow transition layer is always present was demonstrated theoretically and experimentally. Even for atomically flat surfaces where the transition layer is formed by spill-out electrons, collisionless radiative losses turn out to be comparable to or even higher than Joule losses in a metal at room temperature. The transition layer is much thicker for rough interfaces that strongly increase the rate of radiative losses. Radiative losses reduce the efficiency of plasmonic devices. At the same time, the amplitudes of the SPP fields are strongly enhanced at the ENZ region above the metal surface that increases the sensitivity of the detection of biomolecules. The observation of the shift of SPP resonance under an applied electric field may serve as a unique method for studying the electrodynamics of interfaces at the atomic scale.
[1] W. Cai and V. Shalaev, Optical Metamaterials: Fundamentals and Applications (Springer, New York, 2010).

[2] Editorial, A small world full of opportunities, Nat. Mater. 9, 181 (2010).

[3] Editorial, Not so small, Nat. Photonics 8, 877 (2014).

[4] J. A. Schuller, E. S. Barnard, W. Cai, Y. Ch. Jun, J. S. White, and M. L. Brongersma, Plasmonics for extreme light concentration and manipulation, Nat. Mater. 9, 193 (2010).

[5] M. I. Stockman, Nanoplasmonics: Past, present, and glimpse into future, Opt. Express 19, 22029 (2011).

[6] P. Ginzburg, A. Krasavin, Y. Sonnefraud, A. Murphy, R. J. Pollard, S. A. Maier, and A. V. Zayats, Nonlinearly coupled localized plasmon resonances: Resonant second-harmonic generation, Phys. Rev. B 86, 085422 (2012).

[7] J. Khurgin, W.-Y. Tsai, D. P. Tsai, and G. Sun, Landau damping and limit to field confinement and enhancement in plasmonic dimers, ACS Photonics 4, 2871 (2017).

[8] J. Khurgin, How to deal with the loss in plasmonics and metamaterials, Nat. Nanotechnol. 10, 2 (2015).

[9] G. Sun, J. Khurgin, and R. Soref, Practicable enhancement of spontaneous emission using surface plasmons, Appl. Phys. Lett. 90, 111107 (2007).

[10] G. Gumbs, A. Iurov, D. Huang, and W. Pan, Tunable surface plasmon instability leading to emission of radiation, J. Appl. Phys. 118, 054303 (2015).

[11] A. Brandstetter-Kunc, G. Weick, C. A. Downing, D. Weinmann, and R. A. Jalabert, Nonradiative limitations to plasmon propagation in chains of metallic nanoparticles, Phys. Rev. B 94, 205432 (2016).
[12] S. Raza, G. Toscano, A.-P. Jauho, M. Wubs, and N. A. Mortensen, Unusual resonances in nanoplasmonic structures due to nonlocal response, Phys. Rev. B 84, 121412(R) (2011).

[13] N. A. Mortensen, S. Raza, M. Wubs, T. Søndergaard, and S. I. Bozhevolnyi, A generalized non-local optical response theory for plasmonic nanostructures, Nat. Commun. 5, 3809 (2014).

[14] M. Wubs and N. A. Mortensen, Nonlocal response in plasmonic nanostructures, in Quantum Plasmonics, edited by S. I. Bozhevolnyi, L. Martin-Moreno, and F. Garcia-Vidal, Springer Series in Solid-State Sciences Vol. 185 (Springer, Cham, 2017).

[15] M. S. Tame, K. R. McEnery, S. K. Özdemir, J. Lee, S. A. Maier, and M. S. Kim, Quantum plasmonics, Nat. Phys. 9, 329 (2013).

[16] P. A. D. Gonçalves, T. Christensen, N. Rivera, A.-P. Jauho, N. A. Mortensen, and M. Soljačić, Plasmon-emitter interactions at the nanoscale, Nat. Commun. 11, 366 (2020).

[17] A. V. Zayats, I. I. Smolyaninov, and A. A. Maradudin, Nanooptics of surface plasmon polaritons, Phys. Rep. 408, 131 (2005).

[18] N. S. Mueller, Y. Okamura, B. G. M. Vieira, S. Juergensen, H. Lange, E. B. Barros, F. Schulz, and S. Reich, Deep strong light-matter coupling in plasmonic nanoparticle crystals, Nature (London) 583, 780 (2020).

[19] S. V. Boriskina, T. A. Cooper, L. Zeng, G. Ni, J. K. Tong, Yo. Tsurimaki, Y. Huang, L. Meroueh, G. Mahan, and G. Chen, Losses in plasmonics: From mitigating energy dissipation to embracing loss-enabled functionalities, Adv. Opt. Photonics 9, 775 (2017) 
[20] Yu. A. Akimov and H. S. Chu, Plasmon coupling effect on propagation of surface plasmon polaritons at a continuous metal/dielectric interface, Phys. Rev. B 83, 165412 (2011).

[21] L. D. Landau, E. M. Lifshitz, and L. P. Pitaevskii, Electrodynamics of Continuous Media, 2nd ed., Course of Theoretical Physics Vol. 8 (Pergamon, New York, 1984).

[22] N. D. Lang and W. Kohn, Theory of metal surfaces: Charge density and surface energy, Phys. Rev. B 1, 4555 (1970).

[23] C. Cirac, J. B. Pendry, and D. R. Smith, Hydrodynamic model for plasmonics: A macroscopic approach to a microscopic problem, ChemPhysChem 14, 1109 (2013).

[24] See Supplemental Material at http://link.aps.org/supplemental/ 10.1103/PhysRevB.103.L161404 for the solution of the integral equation, details of the experiment, and calculations of charge distribution.

[25] N. M. Litchinitser, A. I. Maimistov, I. R. Gabitov, R. Z. Sagdeev, and V. M. Shalaev, Metamaterials: Electromagnetic enhancement at zero-index transition, Opt. Lett. 33, 2350 (2008).

[26] G. Shvets and X. Li, Theory of laser wakes in plasma channels, Phys. Plasmas 6, 591 (1999).

[27] I. B. Denysenko, S. Ivko, A. Smolyakov, and N. A. Azarenkov, Transmission of electromagnetic waves through a two-layer plasma structure with spatially nonuniform electron density, Phys. Rev. E 86, 056402 (2012).

[28] M. I. Stockman, K. Kneipp, S. I. Bozhevolnyi, S. Saha, A. Dutta, J. Ndukaife, N. Kinsey, H. Reddy, U. Guler, V. M. Shalaev et al., Roadmap on plasmonics, J. Opt. 20, 043001 (2018).

[29] S. Raza, S. I. Bozhevolnyi, M. Wubs, and N. Asger Mortensen, Nonlocal optical response in metallic nanostructures, J. Phys.: Condens. Matter 27, 183204 (2015).
[30] J. B. Khurgin and G. Sun, Impact of surface collisions on enhancement and quenching of the luminescence near the metal nanoparticles, Opt. Express 23, 30739 (2015).

[31] C. Kunz, Messung charakteristischer Energieverluste von Elektronen an leichtoxydierbaren Metallen im Ultrahochvakuum, Z. Phys. 196, 311 (1966).

[32] A. J. Bennett, Influence of the electron charge distribution on surface-plasmon dispersion, Phys. Rev. B 1, 203 (1970).

[33] S. Dhara, B. Sundaravel, T. R. Ravindran, K. G. M. Nair, C. David, B. K. Panigrahi, P. Magudapathy, and K. H. Chen, "Spillout" effect in gold nanoclusters embedded in c$\mathrm{Al}_{2} \mathrm{O}_{3}$ (0001) matrix, Chem. Phys. Lett. 399, 354 (2004).

[34] Yu. A. Akimov, Impact of nonuniform electron density on plasmonic properties of metal nanoparticles, Plasmonics 7, 495 (2012).

[35] A. Campos, N. Troc, E. Cottancin, M. Pellarin, H.-Ch. Weissker, J. Lermé, M. Kociak, and M. Hillenkamp, Plasmonic quantum size effects in silver nanoparticles are dominated by interfaces and local environments, Nat. Phys. 15, 275 (2019).

[36] S. Raza, N. Stengera, S. Kadkhodazadeh, S. V. Fischer, N. Kostesha, A.-P. Jauho, A. Burrows, M. Wubs, and N. Asger Mortensen, Blueshift of the surface plasmon resonance in silver nanoparticles studied with EELS, Nanophotonics 2, 131 (2013).

[37] P. Berini, Plasmon-polariton waves guided by thin lossy metal films of finite width: Bound modes of asymmetric structures, Phys. Rev. B 63, 125417 (2001).

[38] C. David and F. J. García de Abajo, Surface plasmon dependence on the electron density profile at metal surfaces, ACS Nano 8, 9558 (2014). 